\title{
Management of men with a first episode of acute urinary retention due to benign prostatic enlargement
}

\author{
A M Abeygunasekera', S de Silva ${ }^{2}$, A Gurusingha ${ }^{2}$ and S Wijeratne \\ (Index words: Trial without catheter, a adrenoceptor blockers)
}

\begin{abstract}
Objective To determine the effect of the existing management protocol of patients presenting with acute urinary retention due to benign prostatic enlargement on clinical efficacy and surgical practice.
\end{abstract}

Design Prospective study

Setting The Urology Unit at the Teaching Hospital, Karapitiya, Galle.

Patients 100 consecutive patients with a first episode of acute urinary retention due to a clinically benign enlarged prostate.

Measurements Success of voiding urine after one week of treatment with an a adrenoceptor blocker (prazosin). Incidence of subsequent urinary retention during the follow up period of 6 months despite continuing treatment with the drug.

Results Of the 94 patients who completed the follow up period of 6 months, 36 voided successfully after the initial trial without catheter at one week. However, 12 of them developed urinary retention during the follow up and required surgery.

Conclusion Treatment with an a adrenoceptor blocker followed by a single trial without catheter can avoid prostatic surgery in $40 \%$ of patients with acute urinary retention due to benign prostatic enlargement.

\section{Introduction}

Benign prostatic enlargement is common among older men and about 2 to $4 \%$ of them develop acute urinary retention (1). Acute urinary retention (AUR) is initially treated by catheterisation. Transurethral or open prostatectomy is regarded as the definitive treatment for such patients, but due to paucity of expertise and resources in Sri Lanka, such patients are given one or more trials without catheter before scheduling for surgery or referral to a specialised centre. Even in the UK studies have shown that waiting time for transurethral resection of the prostate is unacceptably long (2).

Unfortunately, there are no clear guidelines for management of acute urinary rentenion in the Sri Lankan population, and studies done in this regard are limited. Recently there has been evidence that medical therapy with an a adrenoceptor blocker (prazosin, terazosin) or a 5 a reduc- tase inhibitor (finasteride) is helpful in the management of bladder outflow obstruction caused by benign prostatic enlargement $(3,4)$. Hence the present policy of managing patients with actue urinary retention due to benign prostatic enlargement at the Teaching Hospital, Karapitiya, is to give them a trial without catheter (TWOC) one week after commencing treatment with an a adrenergic blocker. The patients who fail to void after the TWOC are scheduled for surgery and others are given long term therapy with the a blocker. A prospective study was done to determine the effectiveness of this management protocol.

\section{Patients and methods}

Between May and October 2000, 100 consecutive patients admitted with a first AUR having a clinically benign enlarged prostate were included in this study. Initial patient assessment was made by one the authors (AMA) in all cases and the data were recorded. The collected data included the patient demographics, duration of any preceding lower urinary tract symptoms and the size of the prostate assessed by digital rectal examination.

All these patients were started on prazosin and a TWOC was done after one week. Patients who voided successfully were advised to continue treatment with prazosin and to attend the outpatient clinic monthly. The patients who developed urinary retention after the TWOC were scheduled for surgery. All patients were followed up at least for 6 months at the outpatient clinic and their progress was recorded.

\section{Results}

Of the 100 patients, 6 did not come for follow up even after postal reminders. The data of the remaining 94 patients were analysed. The mean age was 68 years (range 55 to 81 years). Of the 94 patients, $56(60 \%)$ voided successfully after the TWOC. 38 patients failed to void after the TWOC and underwent surgery. None of them developed urinary retention during the follow up period after surgery. Twelve of the 56 patients who voided initially, developed urinary retention during the follow up and underwent surgery. Eight of them developed AUR within the first two weeks. Another two patients underwent transurethral resection for severe lower urinary tract symptoms despite treatment with prazosin during the follow up period. 
Sixty seven patients $(71 \%)$ had a prostate gland smaller than $20 \mathrm{~g}$ on clinical assessment. Only 6 patients had a prostate gland larger than $35 \mathrm{~g}$. 33 patients with a prostate gland smaller than $20 \mathrm{~g}$ had a successful TWOC after medical treatment. Eighty two patients $(85 \%)$ had preceding lower urinary tract symptoms for more than 4 weeks before the episode of ARU.

\section{Discussion}

Surgery was the mainstay in treatment for bladder outflow obstruction due to benign prostatic enlargement until recently. Now pharmacological management has become effective with the development of a adrenergic blockers and $5 \mathbf{0}$ (reductase inhibitors. According to previous studies, by adopting a TWOC once, about $20 \%$ of patients with ARU may avoid surgery $(5,6,7)$. In our study, when this was combined with pharmacological therapy the success rate was $40 \%$. Blockade of the a adrenergic receptors in the bladder neck and prostate may have relexed the bladder smooth muscle sufficiently to give a higher rate of successful voiding (4). When the large number of patients who present with ARU to the hospitals is considered, this would save a significant amount of operation time and other resources, and reduce waiting time of deserving patients.

According to previous studies about half of men who initially void successfully will experience AUR within a week and $68 \%$ will experience AUR within a year (8). In our study only $21 \%$ developed AUR over the follow up period of 6 months. The fact that they continued talcing the pharmacological treatment is likely to have contributed to this low AUR rate.

A large proportion $(71 \%)$ of patients in the study had prostates smaller than $20 \mathrm{~g}$ when assessed clinically. Small prostates are known to yield successful TWOC more commonly (9). This could be another reason for the better outcome after the TWOC in our study.

A short or absent history of lower urinary tract symptoms before AUR is more likely to produce a successful TWOC, although some refute it $(5,6,10)$. In our study, only a small number of patients had preceding lower urinary tract symptoms.

The management protocol of patients with AUR at the Urology Unit, Teaching Hospital Karapitiya, seems satisfactory in regard to clinical efficacy and good surgical practice. It could be adopted by other units in the country to save operating time, resources and waiting time for deserving patients.

\section{References}

1. McConnell JD, Bruskevitz R, Walsh P, Andriole G, Lieber M, et al. The effect of finasteride on the risk of acute urinary retention and the need for surgical treatment among men with benign prostatic hyperplasia. New England Journal of Medicine 1998; 338: 557-63.

2. Modi P, Pleat J, Cheetham P, Senior J, Johon R, Fellows GJ. A 23-year review of the management of acute retention of urine: progressing or regressing?. Annals of the Royal College of Surgeons of England 2000; 82: 333-5.

3. Jardin A, Bensadoan H, Delauche-Cavalier MC, Ahali P, BPH - ALF group. Alfuzosin for treatment of benign prostatic hypertrophy. Lancet 1991; 337:1457-61.

4. Osterling JE, Wood AJJ. Benign prostatic hyperplasia: medical and minimally invasive treatment options. New England Journal of Medicine 1995; 332: 99-109.

5. Choong S, Emberton M. Acute urinary retention. British Journal of Urology 2000; 85:186-201.

6. Taube M, Gajraj H. Trial without catheter following acute retention of urine. British Journal of Urology 1989;63:180-2.

7. Murray K. Massey A, Feneley RCL. Acute urinary retention urodynamic assessment. British Journal of Urology 1987; 56: 468-73.

8. Emberton M. Anson K. Acute urinary retention in men: an age old problem. British Medical Journal 1999; 318:921-5.

9. Pickard R, Emberton M, Neal DE. The management of men with acute urinary retention. British Journal of Urology 1998; 81:712-20.

10. Hastre KJ, Dickinson AJ, Ahmad R, Moisey CU. Acute retention of urine: is trial without catheter justified? Journal of the Royal College of Surgeons of Edinburgh 1990; 35: 225-7. 